\title{
Search for Heavy Long-Lived Particles that Decay to Photons at CDF II
}

A. Abulencia, ${ }^{24}$ J. Adelman, ${ }^{13}$ T. Affolder, ${ }^{10}$ T. Akimoto, ${ }^{55}$ M. G. Albrow, ${ }^{17}$ S. Amerio, ${ }^{43}$ D. Amidei,${ }^{35}$ A. Anastassov,${ }^{52}$ K. Anikeev, ${ }^{17}$ A. Annovi, ${ }^{19}$ J. Antos,${ }^{14}$ M. Aoki,${ }^{55}$ G. Apollinari,,${ }^{17}$ T. Arisawa,${ }^{57}$ A. Artikov, ${ }^{15}$ W. Ashmanskas, ${ }^{17}$ A. Attal,${ }^{3}$ A. Aurisano, ${ }^{42}$ F. Azfar, ${ }^{42}$ P. Azzi-Bacchetta, ${ }^{43}$ P. Azzurri,${ }^{46}$ N. Bacchetta, ${ }^{43}$ W. Badgett,${ }^{17}$ A. Barbaro-Galtieri, ${ }^{29}$ V.E. Barnes, ${ }^{48}$ B. A. Barnett, ${ }^{25}$ S. Baroiant,${ }^{7}$ V. Bartsch, ${ }^{31}$ G. Bauer, ${ }^{33}$ P.-H. Beauchemin, ${ }^{34}$ F. Bedeschi, ${ }^{46}$ S. Behari, ${ }^{25}$ G. Bellettini, ${ }^{46}$ J. Bellinger, ${ }^{59}$ A. Belloni, ${ }^{33}$ D. Benjamin, ${ }^{16}$ A. Beretvas, ${ }^{17}$ J. Beringer, ${ }^{29}$ T. Berry, ${ }^{30}$ A. Bhatti, ${ }^{50}$ M. Binkley, ${ }^{17}$ D. Bisello, ${ }^{43}$ I. Bizjak, ${ }^{31}$ R. E. Blair, ${ }^{2}$ C. Blocker, ${ }^{6}$ B. Blumenfeld ${ }^{25}$ A. Bocci, ${ }^{16}$ A. Bodek,${ }^{49}$ V. Boisvert,${ }^{49}$ G. Bolla, ${ }^{48}$ A. Bolshov, ${ }^{33}$ D. Bortoletto,${ }^{48}$ J. Boudreau, ${ }^{47}$ A. Boveia, ${ }^{10}$ B. Brau, ${ }^{10}$ L. Brigliadori, ${ }^{5}$ C. Bromberg, ${ }^{36}$ E. Brubaker ${ }^{13}$ J. Budagov, ${ }^{15}$ H. S. Budd, ${ }^{49}$ S. Budd ${ }^{24}$ K. Burkett, ${ }^{17}$ G. Busetto,${ }^{43}$ P. Bussey, ${ }^{21}$ A. Buzatu, ${ }^{34}$ K. L. Byrum ${ }^{2}$ S. Cabrera, ${ }^{16, \mathrm{q}}$ M. Campanelli, ${ }^{20} \mathrm{M}$. Campbell, ${ }^{35}$ F. Canelli, ${ }^{17}$ A. Canepa,${ }^{45}$ S. Carillo, ${ }^{18, \mathrm{i}}$ D. Carlsmith ${ }^{59}$ R. Carosi, ${ }^{46}$ S. Carron, ${ }^{34}$ B. Casal, ${ }^{11}$ M. Casarsa, ${ }^{54}$ A. Castro, ${ }^{5}$ P. Catastini, ${ }^{46}$ D. Cauz,${ }^{54}$ M. Cavalli-Sforza, ${ }^{3}$ A. Cerri, ${ }^{29}$ L. Cerrito, ${ }^{31, m}$ S. H. Chang ${ }^{28}$ Y. C. Chen, ${ }^{1}$ M. Chertok, ${ }^{7}$ G. Chiarelli, ${ }^{46}$ G. Chlachidze, ${ }^{17}$ F. Chlebana, ${ }^{17}$ I. Cho, ${ }^{28}$ K. Cho,${ }^{28}$ D. Chokheli, ${ }^{15}$ J. P. Chou, ${ }^{22}$ G. Choudalakis, ${ }^{33}$ S. H. Chuang, ${ }^{52}$ K. Chung, ${ }^{12}$ W. H. Chung, ${ }^{59}$ Y. S. Chung, ${ }^{49}$ M. Cilijak, ${ }^{46}$ C. I. Ciobanu, ${ }^{24}$ M. A. Ciocci, ${ }^{46}$ A. Clark, ${ }^{20}$ D. Clark, ${ }^{6}$ M. Coca, ${ }^{16}$ G. Compostella, ${ }^{43}$ M. E. Convery,${ }^{50}$ J. Conway, ${ }^{7}$ B. Cooper,${ }^{31}$ K. Copic, ${ }^{35}$ M. Cordelli, ${ }^{19}$ G. Cortiana,${ }^{43}$ F. Crescioli, ${ }^{46}$ C. Cuenca Almenar, ${ }^{7, q}$ J. Cuevas, ${ }^{11,1}$ R. Culbertson,,${ }^{17}$ J. C. Cully, ${ }^{35}$ S. DaRonco, ${ }^{43}$ M. Datta, ${ }^{17}$ S. D'Auria,${ }^{21}$ T. Davies, ${ }^{21}$ D. Dagenhart, ${ }^{17}$ P. de Barbaro, ${ }^{49}$ S. De Cecco, ${ }^{51}$ A. Deisher, ${ }^{29}$

G. De Lentdecker, ${ }^{49, c}$ G. De Lorenzo, ${ }^{3}$ M. Dell'Orso ${ }^{46}$ F. Delli Paoli, ${ }^{43}$ L. Demortier, ${ }^{50}$ J. Deng, ${ }^{16}$ M. Deninno, ${ }^{5}$ D. De Pedis,${ }^{51}$ P. F. Derwent, ${ }^{17}$ G. P. Di Giovanni, ${ }^{44}$ C. Dionisi, ${ }^{51}$ B. Di Ruzza,${ }^{54}$ J. R. Dittmann, ${ }^{4}$ M. D'Onofrio, ${ }^{3}$ C. Dörr, ${ }^{26}$ S. Donati, ${ }^{46}$ P. Dong, ${ }^{8}$ J. Donini, ${ }^{43}$ T. Dorigo, ${ }^{43}$ S. Dube, ${ }^{52}$ J. Efron, ${ }^{39}$ R. Erbacher, ${ }^{7}$ D. Errede, ${ }^{24}$ S. Errede,${ }^{24}$ R. Eusebi, ${ }^{17}$ H. C. Fang, ${ }^{29}$ S. Farrington, ${ }^{30}$ I. Fedorko,${ }^{46}$ W. T. Fedorko, ${ }^{13}$ R. G. Feild, ${ }^{60}$ M. Feindt, ${ }^{26}$ J. P. Fernandez, ${ }^{32}$ R. Field,${ }^{18}$ G. Flanagan ${ }^{48}$ R. Forrest, ${ }^{7}$ S. Forrester, ${ }^{7}$ M. Franklin, ${ }^{22}$ J. C. Freeman, ${ }^{29}$ I. Furic, ${ }^{13}$ M. Gallinaro, ${ }^{50}$ J. Galyardt, ${ }^{12}$ J. E. Garcia, ${ }^{46}$ F. Garberson, ${ }^{10}$ A. F. Garfinkel, ${ }^{48}$ C. Gay, ${ }^{60}$ H. Gerberich,${ }^{24}$ D. Gerdes,${ }^{35}$ S. Giagu, ${ }^{51}$ P. Giannetti, ${ }^{46}$ K. Gibson, ${ }^{47}$ J. L. Gimmell, ${ }^{49}$ C. Ginsburg, ${ }^{17}$ N. Giokaris, ${ }^{15, a}$ M. Giordani, ${ }^{54}$ P. Giromini, ${ }^{19}$ M. Giunta, ${ }^{46}$ G. Giurgiu, ${ }^{25}$ V. Glagolev, ${ }^{15}$ D. Glenzinski, ${ }^{17}$ M. Gold, ${ }^{37}$ N. Goldschmidt,${ }^{18}$ J. Goldstein, ${ }^{42, b}$ A. Golossanov, ${ }^{17}$ G. Gomez, ${ }^{11}$ G. Gomez-Ceballos, ${ }^{33}$ M. Goncharov ${ }^{53}$ O. González, ${ }^{32}$ I. Gorelov,${ }^{37}$ A. T. Goshaw, ${ }^{16}$ K. Goulianos, ${ }^{50}$ A. Gresele, ${ }^{43}$ S. Grinstein, ${ }^{22}$ C. Grosso-Pilcher, ${ }^{13}$ R. C. Group,${ }^{17}$ U. Grundler, ${ }^{24}$ J. Guimaraes da Costa, ${ }^{22}$ Z. Gunay-Unalan, ${ }^{36}$ C. Haber, ${ }^{29}$ K. Hahn,${ }^{33}$ S. R. Hahn, ${ }^{17}$ E. Halkiadakis, ${ }^{52}$ A. Hamilton ${ }^{20}$ B.-Y. Han,${ }^{49}$ J. Y. Han,${ }^{49}$ R. Handler, ${ }^{59}$

F. Happacher, ${ }^{19}$ K. Hara, ${ }^{55}$ D. Hare,${ }^{52}$ M. Hare, ${ }^{56}$ S. Harper,${ }^{42}$ R. F. Harr, ${ }^{58}$ R. M. Harris, ${ }^{17}$ M. Hartz, ${ }^{47}$ K. Hatakeyama, ${ }^{50}$ J. Hauser, ${ }^{8}$ C. Hays,${ }^{42}$ M. Heck, ${ }^{26}$ A. Heijboer, ${ }^{45}$ B. Heinemann, ${ }^{29}$ J. Heinrich,${ }^{45}$ C. Henderson, ${ }^{33}$ M. Herndon, ${ }^{59}$ J. Heuser, ${ }^{26}$ D. Hidas, ${ }^{16}$ C. S. Hill,,${ }^{10, b}$ D. Hirschbuehl,${ }^{26}$ A. Hocker, ${ }^{17}$ A. Holloway, ${ }^{22}$ S. Hou, ${ }^{1}$ M. Houlden,${ }^{30}$ S.-C. Hsu, ${ }^{9}$ B. T. Huffman, ${ }^{42}$ R. E. Hughes, ${ }^{39}$ U. Husemann, ${ }^{60}$ J. Huston,${ }^{36}$ J. Incandela, ${ }^{10}$ G. Introzzi, ${ }^{46}$ M. Iori, ${ }^{51}$ A. Ivanov, ${ }^{7}$ B. Iyutin, ${ }^{33}$ E. James, ${ }^{17}$ D. Jang, ${ }^{52}$ B. Jayatilaka, ${ }^{16}$ D. Jeans,${ }^{51}$ E. J. Jeon, ${ }^{28}$ S. Jindariani, ${ }^{18}$ W. Johnson, ${ }^{7}$ M. Jones,${ }^{48}$ K. K. Joo, ${ }^{28}$ S. Y. Jun, ${ }^{12}$ J. E. Jung, ${ }^{28}$ T. R. Junk,${ }^{24}$ T. Kamon, ${ }^{53}$ P. E. Karchin, ${ }^{58}$ Y. Kato ${ }^{41}$ Y. Kemp,${ }^{26}$ R. Kephart, ${ }^{17}$

U. Kerzel, ${ }^{26}$ V. Khotilovich, ${ }^{53}$ B. Kilminster, ${ }^{39}$ D. H. Kim, ${ }^{28}$ H. S. Kim, ${ }^{28}$ J. E. Kim, ${ }^{28}$ M. J. Kim, ${ }^{17}$ S. B. Kim, ${ }^{28}$ S. H. Kim,${ }^{55}$ Y. K. Kim, ${ }^{13}$ N. Kimura, ${ }^{55}$ L. Kirsch, ${ }^{6}$ S. Klimenko, ${ }^{18}$ M. Klute, ${ }^{33}$ B. Knuteson, ${ }^{33}$ B. R. Ko, ${ }^{16}$ K. Kondo,${ }^{57}$ D. J. Kong, ${ }^{28}$ J. Konigsberg, ${ }^{18}$ A. Korytov, ${ }^{18}$ A. V. Kotwal, ${ }^{16}$ A. C. Kraan, ${ }^{45}$ J. Kraus,${ }^{24}$ M. Kreps, ${ }^{26}$ J. Kroll, ${ }^{45}$ N. Krumnack, ${ }^{4}$ M. Kruse, ${ }^{16}$ V. Krutelyov, ${ }^{10}$ T. Kubo, ${ }^{55}$ S. E. Kuhlmann, ${ }^{2}$ T. Kuhr ${ }^{26}$ N. P. Kulkarni, ${ }^{58}$ Y. Kusakabe, ${ }^{57}$ S. Kwang, ${ }^{13}$ A. T. Laasanen, ${ }^{48}$ S. Lai, ${ }^{34}$ S. Lami,${ }^{46}$ S. Lammel, ${ }^{17}$ M. Lancaster, ${ }^{31}$ R. L. Lander, ${ }^{7}$ K. Lannon, ${ }^{39}$ A. Lath, ${ }^{52}$ G. Latino, ${ }^{46}$ I. Lazzizzera, ${ }^{43}$ T. LeCompte, ${ }^{2}$ E. Lee,${ }^{53}$ J. Lee, ${ }^{49}$ J. Lee, ${ }^{28}$ Y. J. Lee ${ }^{28}$ S. W. Lee, ${ }^{53, o}$ R. Lefèvre, ${ }^{20}$ N. Leonardo, ${ }^{33}$ S. Leone, ${ }^{46}$ S. Levy, ${ }^{13}$ J. D. Lewis,${ }^{17}$ C. Lin, ${ }^{60}$ C. S. Lin, ${ }^{17}$ M. Lindgren, ${ }^{17}$ E. Lipeles, ${ }^{9}$ A. Lister, ${ }^{7}$ D. O. Litvintsev, ${ }^{17}$ T. Liu, ${ }^{17}$ N. S. Lockyer, ${ }^{45}$ A. Loginov, ${ }^{60}$ M. Loreti, ${ }^{43}$ R.-S. Lu, ${ }^{1}$ D. Lucchesi, ${ }^{43}$ P. Lujan, ${ }^{29}$ P. Lukens, ${ }^{17}$ G. Lungu, ${ }^{18}$ L. Lyons, ${ }^{42}$ J. Lys, ${ }^{29}$ R. Lysak, ${ }^{14}$ E. Lytken, ${ }^{48}$ P. Mack,${ }^{26}$ D. MacQueen, ${ }^{34}$ R. Madrak,${ }^{17}$ K. Maeshima, ${ }^{17}$

K. Makhoul, ${ }^{33}$ T. Maki, ${ }^{23}$ P. Maksimovic, ${ }^{25}$ S. Malde, ${ }^{42}$ S. Malik, ${ }^{31}$ G. Manca,${ }^{30}$ F. Margaroli, ${ }^{5}$ R. Marginean, ${ }^{17}$ C. Marino, ${ }^{26}$ C. P. Marino, ${ }^{24}$ A. Martin, ${ }^{60}$ M. Martin, ${ }^{25}$ V. Martin, ${ }^{21, g}$ M. Martínez, ${ }^{3}$ R. Martínez-Ballarín, ${ }^{32}$

T. Maruyama ${ }^{55}$ P. Mastrandrea, ${ }^{51}$ T. Masubuchi, ${ }^{55}$ H. Matsunaga, ${ }^{55}$ M. E. Mattson, ${ }^{58}$ R. Mazini, ${ }^{34}$ P. Mazzanti, ${ }^{5}$ K. S. McFarland, ${ }^{49}$ P. McIntyre ${ }^{53}$ R. McNulty, ${ }^{30, f}$ A. Mehta ${ }^{30}$ P. Mehtala, ${ }^{23}$ S. Menzemer, ${ }^{11, i}$ A. Menzione, ${ }^{46}$ P. Merkel, ${ }^{48}$ C. Mesropian, ${ }^{50}$ A. Messina, ${ }^{36}$ T. Miao, ${ }^{17}$ N. Miladinovic, ${ }^{6}$ J. Miles, ${ }^{33}$ R. Miller,${ }^{36}$ C. Mills, ${ }^{10}$ M. Milnik,${ }^{26}$ A. Mitra, ${ }^{1}$ G. Mitselmakher ${ }^{18}$ A. Miyamoto, ${ }^{27}$ S. Moed,${ }^{20}$ N. Moggi, ${ }^{5}$ B. Mohr, ${ }^{8}$ C. S. Moon, ${ }^{28}$ R. Moore,${ }^{17}$ M. Morello, ${ }^{46}$ P. Movilla Fernandez, ${ }^{29}$ J. Mülmenstädt,${ }^{29}$ A. Mukherjee, ${ }^{17}$ Th. Muller, ${ }^{26}$ R. Mumford, ${ }^{25}$ P. Murat, ${ }^{17}$ M. Mussini, ${ }^{5}$ 
J. Nachtman, ${ }^{17}$ A. Nagano, ${ }^{55}$ J. Naganoma,${ }^{57}$ K. Nakamura,${ }^{55}$ I. Nakano, ${ }^{40}$ A. Napier,${ }^{56}$ V. Necula, ${ }^{16}$ C. Neu, ${ }^{45}$ M. S. Neubauer, ${ }^{9}$ J. Nielsen, ${ }^{29, n}$ L. Nodulman, ${ }^{2}$ O. Norniella, ${ }^{3}$ E. Nurse, ${ }^{31}$ S. H. Oh, ${ }^{16}$ Y. D. Oh, ${ }^{28}$ I. Oksuzian, ${ }^{18}$

T. Okusawa,${ }^{41}$ R. Oldeman, ${ }^{30}$ R. Orava,${ }^{23}$ K. Osterberg, ${ }^{23}$ C. Pagliarone,${ }^{46}$ E. Palencia, ${ }^{11}$ V. Papadimitriou, ${ }^{17}$ A. Papaikonomou, ${ }^{26}$ A. A. Paramonov,${ }^{13}$ B. Parks, ${ }^{39}$ S. Pashapour,,${ }^{34}$ J. Patrick, ${ }^{17}$ G. Pauletta, ${ }^{54}$ M. Paulini, ${ }^{12}$ C. Paus,${ }^{33}$ D. E. Pellett,${ }^{7}$ A. Penzo, ${ }^{54}$ T. J. Phillips,${ }^{16}$ G. Piacentino, ${ }^{46}$ J. Piedra, ${ }^{44}$ L. Pinera,${ }^{18}$ K. Pitts,${ }^{24}$ C. Plager, ${ }^{8}$ L. Pondrom,${ }^{59}$ X. Portell, ${ }^{3}$ O. Poukhov, ${ }^{15}$ N. Pounder, ${ }^{42}$ F. Prakoshyn,,${ }^{15}$ A. Pronko, ${ }^{17}$ J. Proudfoot, ${ }^{2}$ F. Ptohos, ${ }^{19,}$, G. Punzi, ${ }^{46}$ J. Pursley, ${ }^{25}$ J. Rademacker, ${ }^{42, \mathrm{~b}}$ A. Rahaman, ${ }^{47}$ V. Ramakrishnan, ${ }^{59}$ N. Ranjan, ${ }^{48}$ I. Redondo, ${ }^{32}$ B. Reisert, ${ }^{17}$ V. Rekovic, ${ }^{37}$ P. Renton, ${ }^{42}$ M. Rescigno, ${ }^{51}$ S. Richter, ${ }^{26}$ F. Rimondi, ${ }^{5}$ L. Ristori, ${ }^{46}$ A. Robson, ${ }^{21}$ T. Rodrigo, ${ }^{11}$ E. Rogers,${ }^{24}$ S. Rolli, ${ }^{56}$ R. Roser, ${ }^{17}$ M. Rossi ${ }^{54}$ R. Rossin,${ }^{10}$ P. Roy,${ }^{34}$ A. Ruiz, ${ }^{11}$ J. Russ, ${ }^{12}$ V. Rusu, ${ }^{13}$ H. Saarikko, ${ }^{23}$ A. Safonov, ${ }^{53}$ W. K. Sakumoto, ${ }^{49}$ G. Salamanna, ${ }^{51}$ O. Saltó, ${ }^{3}$ L. Santi, ${ }^{54}$ S. Sarkar,${ }^{51}$ L. Sartori, ${ }^{46}$ K. Sato, ${ }^{17}$ P. Savard,${ }^{34}$ A. Savoy-Navarro,${ }^{44}$ T. Scheidle,${ }^{26}$ P. Schlabach, ${ }^{17}$ E. E. Schmidt, ${ }^{17}$ M. P. Schmidt,${ }^{60}$ M. Schmitt, ${ }^{38}$ T. Schwarz, ${ }^{7}$ L. Scodellaro, ${ }^{11}$ A. L. Scott, ${ }^{10}$ A. Scribano, ${ }^{46}$ F. Scuri, ${ }^{46}$ A. Sedov, ${ }^{48}$ S. Seidel, ${ }^{37}$ Y. Seiya, ${ }^{41}$ A. Semenov, ${ }^{15}$ L. Sexton-Kennedy, ${ }^{17}$ A. Sfyrla, ${ }^{20}$ S.Z. Shalhout, ${ }^{58}$ M. D. Shapiro, ${ }^{29}$ T. Shears, ${ }^{30}$ P. F. Shepard, ${ }^{47}$ D. Sherman, ${ }^{22}$ M. Shimojima, ${ }^{55, k}$ M. Shochet, ${ }^{13}$ Y. Shon, ${ }^{59}$ I. Shreyber, ${ }^{20}$ A. Sidoti, ${ }^{46}$ P. Sinervo, ${ }^{34}$ A. Sisakyan, ${ }^{15}$ A. J. Slaughter, ${ }^{17}$ J. Slaunwhite, ${ }^{39}$ K. Sliwa, ${ }^{56}$ J. R. Smith, ${ }^{7}$ F. D. Snider, ${ }^{17}$ R. Snihur, ${ }^{34}$ M. Soderberg, ${ }^{35}$ A. Soha,${ }^{7}$ S. Somalwar, ${ }^{52}$ V. Sorin,${ }^{36}$ J. Spalding, ${ }^{17}$ F. Spinella, ${ }^{46}$ T. Spreitzer, ${ }^{34}$ P. Squillacioti, ${ }^{46}$ M. Stanitzki, ${ }^{60}$ A. Staveris-Polykalas, ${ }^{46}$ R. St. Denis, ${ }^{21}$ B. Stelzer, ${ }^{8}$ O. Stelzer-Chilton, ${ }^{42}$ D. Stentz, ${ }^{38}$ J. Strologas, ${ }^{37}$ D. Stuart, ${ }^{10}$ J. S. Suh,${ }^{28}$ A. Sukhanov, ${ }^{18}$ H. Sun,${ }^{56}$ I. Suslov, ${ }^{15}$ T. Suzuki, ${ }^{55}$ A. Taffard, ${ }^{24, p}$ R. Takashima ${ }^{40}$ Y. Takeuchi, ${ }^{55}$ R. Tanaka, ${ }^{40}$ M. Tecchio, ${ }^{35}$ P. K. Teng, ${ }^{1}$ K. Terashi, ${ }^{50}$ J. Thom, ${ }^{17, d}$ A. S. Thompson, ${ }^{21}$ E. Thomson, ${ }^{45}$ P. Tipton, ${ }^{60}$ V. Tiwari, ${ }^{12}$ S. Tkaczyk,${ }^{17}$ D. Toback ${ }^{53}$ S. Tokar,${ }^{14}$ K. Tollefson,${ }^{36}$ T. Tomura,${ }^{55}$ D. Tonelli, ${ }^{46}$ S. Torre,${ }^{19}$ D. Torretta, ${ }^{17}$ S. Tourneur, ${ }^{44} \mathrm{~W}$. Trischuk, ${ }^{34} \mathrm{~S}$. Tsuno, ${ }^{40} \mathrm{Y}$. Tu, ${ }^{45} \mathrm{~N}$. Turini,${ }^{46} \mathrm{~F}$. Ukegawa, ${ }^{55} \mathrm{~S}$. Uozumi,${ }^{55} \mathrm{~S}$. Vallecorsa, ${ }^{20}$ N. van Remortel, ${ }^{23}$ A. Varganov, ${ }^{35}$ E. Vataga,${ }^{37}$ F. Vazquez, ${ }^{18, \mathrm{i}} \mathrm{G}$. Velev, ${ }^{17} \mathrm{G}$. Veramendi, ${ }^{24}$ V. Veszpremi, ${ }^{48} \mathrm{M}$. Vidal,${ }^{32}$ R. Vidal, ${ }^{17}$ I. Vila, ${ }^{11}$ R. Vilar, ${ }^{11}$ T. Vine, ${ }^{31}$ I. Vollrath, ${ }^{34}$ I. Volobouev, ${ }^{29, o}$ G. Volpi,${ }^{46}$ F. Würthwein, ${ }^{9}$ P. Wagner, ${ }^{53}$ R. G. Wagner, ${ }^{2}$ R. L. Wagner, ${ }^{17}$ J. Wagner,${ }^{26}$ W. Wagner,${ }^{26}$ R. Wallny, ${ }^{8}$ S. M. Wang, ${ }^{1}$ A. Warburton, ${ }^{34}$ D. Waters, ${ }^{31}$ M. Weinberger, ${ }^{53}$ W. C. Wester III, ${ }^{17}$ B. Whitehouse, ${ }^{56}$ D. Whiteson, ${ }^{45}$ A. B. Wicklund, ${ }^{2}$ E. Wicklund, ${ }^{17}$ G. Williams, ${ }^{34}$ H. H. Williams, ${ }^{45}$ P. Wilson, ${ }^{17}$ B. L. Winer, ${ }^{39}$ P. Wittich, ${ }^{17, d}$ S. Wolbers, ${ }^{17}$ C. Wolfe, ${ }^{13}$ T. Wright,${ }^{35}$ X. Wu, ${ }^{20}$

S. M. Wynne, ${ }^{30}$ A. Yagil, ${ }^{9}$ K. Yamamoto, ${ }^{41}$ J. Yamaoka, ${ }^{52}$ T. Yamashita ${ }^{40}$ C. Yang, ${ }^{60}$ U. K. Yang, ${ }^{13, j}$ Y. C. Yang, ${ }^{28}$ W. M. Yao, ${ }^{29}$ G. P. Yeh, ${ }^{17}$ J. Yoh, ${ }^{17}$ K. Yorita, ${ }^{13}$ T. Yoshida, ${ }^{41}$ G. B. Yu, ${ }^{49}$ I. Yu, ${ }^{28}$ S. S. Yu, ${ }^{17}$ J. C. Yun, ${ }^{17}$ L. Zanello, ${ }^{51}$ A. Zanetti, ${ }^{54}$ I. Zaw, ${ }^{22}$ X. Zhang, ${ }^{24}$ J. Zhou, ${ }^{52}$ and S. Zucchelli ${ }^{5}$

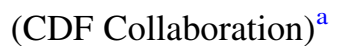

\author{
${ }^{1}$ Institute of Physics, Academia Sinica, Taipei, Taiwan 11529, Republic of China \\ ${ }^{2}$ Argonne National Laboratory, Argonne, Illinois 60439, USA \\ ${ }^{3}$ Institut de Fisica d'Altes Energies, Universitat Autonoma de Barcelona, E-08193, Bellaterra (Barcelona), Spain \\ ${ }^{4}$ Baylor University, Waco, Texas 76798, USA \\ ${ }^{5}$ Istituto Nazionale di Fisica Nucleare, University of Bologna, I-40127 Bologna, Italy \\ ${ }^{6}$ Brandeis University, Waltham, Massachusetts 02254, USA \\ ${ }^{7}$ University of California, Davis, Davis, California 95616, USA \\ ${ }^{8}$ University of California, Los Angeles, Los Angeles, California 90024, USA \\ ${ }^{9}$ University of California, San Diego, La Jolla, California 92093, USA \\ ${ }^{10}$ University of California, Santa Barbara, Santa Barbara, California 93106, USA \\ ${ }^{11}$ Instituto de Fisica de Cantabria, CSIC-University of Cantabria, 39005 Santander, Spain \\ ${ }^{12}$ Carnegie Mellon University, Pittsburgh, Pennsylvania 15213, USA \\ ${ }^{13}$ Enrico Fermi Institute, University of Chicago, Chicago, Illinois 60637, USA \\ ${ }^{14}$ Comenius University, 84248 Bratislava, Slovakia; \\ Institute of Experimental Physics, 04001 Kosice, Slovakia \\ ${ }^{15}$ Joint Institute for Nuclear Research, RU-141980 Dubna, Russia \\ ${ }^{16}$ Duke University, Durham, North Carolina 27708, USA \\ ${ }^{17}$ Fermi National Accelerator Laboratory, Batavia, Illinois 60510, USA \\ ${ }^{18}$ University of Florida, Gainesville, Florida 32611, USA \\ ${ }^{19}$ Laboratori Nazionali di Frascati, Istituto Nazionale di Fisica Nucleare, I-00044 Frascati, Italy \\ ${ }^{20}$ University of Geneva, CH-1211 Geneva 4, Switzerland \\ ${ }^{21}$ Glasgow University, Glasgow G12 8QQ, United Kingdom \\ ${ }^{22}$ Harvard University, Cambridge, Massachusetts 02138, USA
}


${ }^{23}$ Division of High Energy Physics, Department of Physics, University of Helsinki and Helsinki Institute of Physics, FIN-00014, Helsinki, Finland

${ }^{24}$ University of Illinois, Urbana, Illinois 61801, USA

${ }^{25}$ The Johns Hopkins University, Baltimore, Maryland 21218, USA

${ }^{26}$ Institut für Experimentelle Kernphysik, Universität Karlsruhe, 76128 Karlsruhe, Germany

${ }^{27}$ High Energy Accelerator Research Organization (KEK), Tsukuba, Ibaraki 305, Japan

${ }^{28}$ Center for High Energy Physics: Kyungpook National University, Taegu 702-701, Korea; Seoul National University, Seoul 151-742, Korea; SungKyunKwan University, Suwon 440-746, Korea

${ }^{29}$ Ernest Orlando Lawrence Berkeley National Laboratory, Berkeley, California 94720, USA

${ }^{30}$ University of Liverpool, Liverpool L69 7ZE, United Kingdom

${ }^{31}$ University College London, London WC1E 6BT, United Kingdom

${ }^{32}$ Centro de Investigaciones Energeticas Medioambientales y Tecnologicas, E-28040 Madrid, Spain

${ }^{33}$ Massachusetts Institute of Technology, Cambridge, Massachusetts 02139, USA

${ }^{34}$ Institute of Particle Physics: McGill University, Montréal, Canada H3A 2T8; and University of Toronto, Toronto, Canada M5S $1 A 7$

${ }^{35}$ University of Michigan, Ann Arbor, Michigan 48109, USA

${ }^{36}$ Michigan State University, East Lansing, Michigan 48824, USA

${ }^{37}$ University of New Mexico, Albuquerque, New Mexico 87131, USA

${ }^{38}$ Northwestern University, Evanston, Illinois 60208, USA

${ }^{39}$ The Ohio State University, Columbus, Ohio 43210, USA

${ }^{40}$ Okayama University, Okayama 700-8530, Japan

${ }^{41}$ Osaka City University, Osaka 588, Japan

${ }^{42}$ University of Oxford, Oxford OX1 3RH, United Kingdom

${ }^{43}$ University of Padova, Istituto Nazionale di Fisica Nucleare, Sezione di Padova-Trento, I-35131 Padova, Italy

${ }^{44}$ LPNHE, Universite Pierre et Marie Curie/IN2P3-CNRS, UMR7585, Paris, F-75252 France

${ }^{45}$ University of Pennsylvania, Philadelphia, Pennsylvania 19104, USA

${ }^{46}$ Istituto Nazionale di Fisica Nucleare Pisa, Universities of Pisa, Siena and Scuola Normale Superiore, I-56127 Pisa, Italy

${ }^{47}$ University of Pittsburgh, Pittsburgh, Pennsylvania 15260, USA

${ }^{48}$ Purdue University, West Lafayette, Indiana 47907, USA

${ }^{49}$ University of Rochester, Rochester, New York 14627, USA

${ }^{50}$ The Rockefeller University, New York, New York 10021, USA

${ }^{51}$ Istituto Nazionale di Fisica Nucleare, Sezione di Roma 1, University of Rome "La Sapienza," I-00185 Roma, Italy

${ }^{52}$ Rutgers University, Piscataway, New Jersey 08855, USA

${ }^{53}$ Texas A\&M University, College Station, Texas 77843, USA

${ }^{54}$ Istituto Nazionale di Fisica Nucleare, University of Trieste/Udine, Italy

${ }^{55}$ University of Tsukuba, Tsukuba, Ibaraki 305, Japan

${ }^{56}$ Tufts University, Medford, Massachusetts 02155, USA

${ }^{57}$ Waseda University, Tokyo 169, Japan

${ }^{58}$ Wayne State University, Detroit, Michigan 48201, USA

${ }^{59}$ University of Wisconsin, Madison, Wisconsin 53706, USA

${ }^{60}$ Yale University, New Haven, Connecticut 06520, USA

(Received 5 April 2007; published 17 September 2007)

We present the first search for heavy, long-lived particles that decay to photons at a hadron collider. We use a sample of $\gamma+$ jet + missing transverse energy events in $p \bar{p}$ collisions at $\sqrt{s}=1.96 \mathrm{TeV}$ taken with the CDF II detector. Candidate events are selected based on the arrival time of the photon at the detector. Using an integrated luminosity of $570 \mathrm{pb}^{-1}$ of collision data, we observe 2 events, consistent with the background estimate of $1.3 \pm 0.7$ events. While our search strategy does not rely on model-specific dynamics, we set cross section limits in a supersymmetric model with $\tilde{\chi}_{1}^{0} \rightarrow \gamma \widetilde{G}$ and place the world-best $95 \%$ C.L. lower limit on the $\tilde{\chi}_{1}^{0}$ mass of $101 \mathrm{GeV} / c^{2}$ at $\tau_{\tilde{\chi}_{1}^{0}}=5 \mathrm{~ns}$.

Searches for events with final state photons and missing transverse energy $\left(\not_{T}\right)$ at collider experiments are sensitive to new physics from a wide variety of models [1] including gauge mediated supersymmetry breaking (GMSB) [2]. In these models the lightest neutralino $\left(\widetilde{\mathcal{X}}_{1}^{0}\right)$ decays into a photon $(\gamma)$ and a weakly interacting, stable gravitino $(\tilde{G})$ that gives rise to $\not_{T}$ by leaving the detector without depositing any energy. The observation of an $e e \gamma \gamma \mathbb{E}_{T}$ candidate event by the CDF experiment during run I at the Fermilab Tevatron [3] has increased the interest in experi- 
mental tests of this class of theories. Most subsequent searches have focused on promptly produced photons $[4,5]$; however, the $\tilde{\chi}_{1}^{0}$ can have a lifetime on the order of nanoseconds or more. This Letter summarizes [6] the first search for heavy, long-lived particles that decay to photons at a hadron collider. The data comprise $570 \pm 34 \mathrm{pb}^{-1}$ of $p \bar{p}$ collisions at $\sqrt{s}=1.96 \mathrm{TeV}$ from the Tevatron collected with the CDF II detector [7]. Previous searches for nanosecond-lifetime particles using nontiming techniques have yielded null results [8].

We optimize our selection requirements using a GMSB model with a standard choice of parameters [9] and vary the values of the $\tilde{\chi}_{1}^{0}$ mass and lifetime. However, the final search strategy is chosen to be sufficiently general and independent of the specific GMSB model dynamics to yield results that are approximately valid for any model producing the same reconstructed final state topology and kinematics [10]. The inclusive GMSB production cross section is dominated by pair production of gauginos. The gauginos decay promptly, resulting in a pair of long-lived $\tilde{\chi}_{1}^{0}$ 's in association with other final state particles that can be identified as jets. For a heavy $\tilde{\chi}_{1}^{0}$ decaying inside the detector, the photon can arrive at the face of the detector with a time delay relative to promptly produced photons. To have good sensitivity for nanosecond-lifetime $\tilde{\chi}_{1}^{0}$ 's [10], we search for events that contain a time-delayed photon, $\mathscr{E}_{T}$, and $\geq 1$ jet.

Here we briefly describe the aspects of the detector relevant to this analysis. The magnetic spectrometer consists of tracking devices inside a 3-m diameter, 5-m long superconducting solenoid magnet that operates at 1.4 T. An eight-layer silicon microstrip detector array and a $3.1-\mathrm{m}$ long drift chamber with 96 layers of sense wires measure the position $\left(\vec{x}_{i}\right)$ and time $\left(t_{i}\right)$ of the $p \bar{p}$ interaction [11] and the momenta of charged particles. Muons from collisions or cosmic rays are identified by a system of drift chambers situated outside the calorimeters in the region with pseudorapidity $|\eta|<1.1$. The calorimeter consists of projective towers with electromagnetic and hadronic compartments. It is divided into a central barrel that surrounds the solenoid coil $(|\eta|<1.1)$ and a pair of end plugs that cover the region $1.1<|\eta|<3$.6. Both calorimeters are used to identify and measure the energy and position of photons, electrons, jets, and $\mathscr{E}_{T}$. The electromagnetic calorimeters were recently instrumented with a new system, the EMTiming system (completed in Fall 2004) [12], that measures the arrival time of electrons and photons in each tower with $|\eta|<2.1$ for all energies above $\sim 5 \mathrm{GeV}$. The system has a fixed-threshold discriminator and a time-to-digital converter (TDC) connected to each electromagnetic tower and has an intrinsic time-of-arrival resolution of $0.6 \mathrm{~ns}$.

The time and position of arrival of the photon at the calorimeter, $t_{f}$ and $\vec{x}_{f}$, are used to separate the photons from the decays of heavy, long-lived $\tilde{\chi}_{1}^{0}$ 's from promptly produced photons or photons from noncollision sources. We define the corrected arrival time of the photon as $t_{c}^{\gamma} \equiv$ $t_{f}-t_{i}-\frac{\left|\vec{x}_{f}-\vec{x}_{i}\right|}{c}$. The $t_{c}^{\gamma}$ distribution for promptly produced, high energy photons is Gaussian with a mean of zero by construction and has a standard deviation that depends only on the measurement resolution assuming that the $p \bar{p}$ production vertex has been correctly identified. Photons from heavy, long-lived particles can have arrival times that are many standard deviations larger than zero.

The analysis preselection is summarized in Table I, with the effect on an example GMSB model point at $m_{\tilde{\chi}_{1}^{0}}=$ $100 \mathrm{GeV} / c^{2}$ and $\tau_{\tilde{\chi}_{1}^{0}}=5 \mathrm{~ns}$ for comparison. It begins with events passing an online, three-level trigger by having a photon candidate in the region $|\eta|<1.1$ with $E_{T}>$ $25 \mathrm{GeV}$ and $\not E_{T}>25 \mathrm{GeV}$. Offline, the highest $E_{T}$ photon candidate in the fiducial region of the calorimeter is required to have $E_{T}>30 \mathrm{GeV}$ and to pass the standard photon identification requirements [4] with a minor modification [13]. We require the event to have $\mathscr{E}_{T}>30 \mathrm{GeV}$ where the trigger is $100 \%$ efficient. We require at least one jet with $\left|\eta^{\text {jet }}\right|<2.0$ and $E_{T}^{\text {jet }}>30 \mathrm{GeV}$ [14]. Since a second photon can be identified as a jet, the analysis is sensitive to signatures where one or both $\tilde{\chi}_{1}^{0}$ 's decay inside the detector. To ensure a high quality $t_{i}$ and $\vec{x}_{i}$ measurement, we require a vertex with at least 4 tracks, $\sum_{\text {tracks }} p_{T}>$ $15 \mathrm{GeV} / c$, and $\left|z_{i}\right|<60 \mathrm{~cm}$; this also helps to reduce noncollision backgrounds. For events with multiple reconstructed vertices, we pick the vertex with the highest $\sum_{\text {tracks }} p_{T}$. To reduce cosmic ray background, events are rejected if there are hits in a muon chamber that are not matched to any track and are within $30^{\circ}$ of the photon. After the above requirements there are 11932 events in the data sample.

TABLE I. The data selection criteria and efficiencies for the example GMSB model point. The efficiencies listed are, in general, model-dependent and have a fractional uncertainty of $10 \%$. Model-independent efficiencies are indicated with an asterisk. The collision fiducial requirement of $\left|z_{i}\right|<60 \mathrm{~cm}$ is part of the good vertex requirement $(95 \%)$ and is estimated from data.

\begin{tabular}{ll}
\hline \hline Requirements & $\begin{array}{c}\text { Cumulative (individual) } \\
\text { efficiency (\%) }\end{array}$ \\
\hline Preselection requirements & $54(54)$ \\
$E_{T}^{\gamma}>30 \mathrm{GeV}, \mathbb{E}_{T}>30 \mathrm{GeV}$ & $39(74)^{*}$ \\
Photon ID and fiducial, $|\eta|<1.0$ & $31(79)$ \\
Good vertex, $\sum_{\text {tracks }} p_{T}>15 \mathrm{GeV} / c$ & $24(77)$ \\
$\left|\eta^{\mathrm{jet}}\right|<2.0, E_{T}^{\mathrm{jet}}>30 \mathrm{GeV}$ & $23(98)^{*}$ \\
Cosmic ray rejection & \\
Requirements after optimization & $21(92)$ \\
$\mathscr{E}_{T}>40 \mathrm{GeV}, E_{T}^{\mathrm{jet}}>35 \mathrm{GeV}$ & $18(86)$ \\
$\Delta \phi\left(\mathbb{E}_{T}\right.$, jet $)>1 \mathrm{rad}$ & $6(33)$ \\
$2 \mathrm{~ns}<t_{c}^{\gamma}<10 \mathrm{~ns}$ & \\
\hline \hline
\end{tabular}


There are two major classes of background events: collision and noncollision photon candidates. Collision photons are presumed to come from standard model interactions, e.g., $\quad \gamma+$ jet + mismeasured $\mathbb{E}_{T}$, dijet + mismeasured $\not{E}_{T}$, where the jet is misidentified as a $\gamma$, and $W \rightarrow e \nu$, where the electron is misidentified as a $\gamma$. Noncollision backgrounds come from cosmic rays and beam effects that can produce photon candidates, $\mathbb{E}_{T}$, and sometimes the reconstructed jet. We separate data events as a function of $t_{c}^{\gamma}$ into several control regions that allow us to estimate the number of background events in the final signal region by fitting to the data using collision and noncollision shapes as shown in Fig. 1.

Collision photons are subdivided in two subclasses: correct and incorrect vertex selection [12]. An incorrect vertex can be selected when two or more collisions occur in one beam bunch crossing, making it possible that the highest reconstructed $\sum_{\text {tracks }} p_{T}$ vertex does not produce the photon. While the fraction of events with incorrect vertices depends on the final event selection criteria, the $t_{c}^{\gamma}$ distribution for each subclass is estimated separately using $W \rightarrow$ $e \nu$ data where the electron track is dropped from the vertexing. For events with a correctly associated vertex, the $t_{c}^{\gamma}$ distribution is Gaussian and centered at zero with a standard deviation of 0.64 ns [12]. For those with an incorrectly selected vertex the $t_{c}^{\gamma}$ distribution is also Gaussian with a standard deviation of $2.05 \mathrm{~ns}$.

The $t_{c}^{\gamma}$ distributions for both noncollision backgrounds are estimated separately from data using events with no reconstructed tracks. Photon candidates from cosmic rays are not correlated in time with collisions, and therefore their $t_{c}^{\gamma}$ distribution is roughly flat. Beam halo photon candidates are produced by muons that originate upstream of the detector (from the $p$ direction) and travel through the calorimeter, typically depositing small amounts of energy. When the muon deposits significant energy in the EM calorimeter, it can be misidentified as a photon and cause

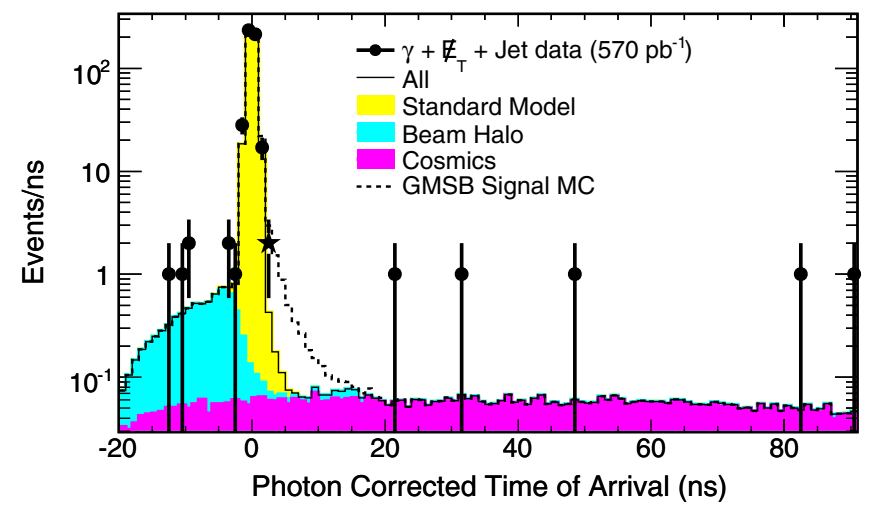

FIG. 1 (color online). The time distribution for photons passing all but the final timing requirement for the background predictions, data (marked with a star for the signal region), and a GMSB signal for the example model point.
$\mathscr{E}_{T}$. These photons populate predominantly the negative $t_{c}^{\gamma}$ region, but can contribute to the signal region.

The background prediction uses control regions outside the signal time window but well within the $132 \mathrm{~ns}$ time window that the calorimeter uses to measure the energy. The noncollision backgrounds are normalized to match the number of events in two time windows: a beam halodominated window at $\{-20,-6\}$ ns, selected to be $3 \sigma$ away from the wrong vertex collision background, and a cosmic rays-dominated window at $\{25,90\} \mathrm{ns}$, well away from the standard model and beam halo contributions. The collision background is estimated by fitting events in the $\{-10,1.2\}$ ns window with the noncollision contribution subtracted and with the fraction of correct to incorrect vertex events allowed to vary. In this way the background for the signal region is entirely estimated from data samples. The systematic uncertainty on the background estimate is dominated by our ability to calibrate the mean of the $t_{c}^{\gamma}$ distribution for prompt photons. We find a variation of 200 ps on the mean and 20 ps on the standard deviation of the distribution by considering various possible event selection criteria. The systematic uncertainty due to these variations is added in quadrature with the statistical uncertainties of the final fit procedure.

We estimate the sensitivity to heavy, long-lived particles that decay to photons using GMSB models for different $\tilde{\chi}_{1}^{0}$ masses and lifetimes. Events from all SUSY processes are simulated with the PYTHIA Monte Carlo program [15] along with the detector simulation [16]. The acceptance is the ratio of simulated events that pass all the requirements to all events produced. It is used in the optimization procedure and in the final limit setting and depends on a number of effects. The fraction of $\tilde{\chi}_{1}^{0}$ decays in the detector volume is the dominant effect on the acceptance. For a given lifetime this depends on the boost of the $\tilde{\chi}_{1}^{0}$. A highly boosted $\tilde{\chi}_{1}^{0}$ that decays in the detector typically does not contribute to the acceptance because it tends to produce a photon traveling in the same direction as the $\tilde{\chi}_{1}^{0}$. Thus, the photon's arrival time is indistinguishable from promptly produced photons. At small boosts the decay is more likely to happen inside the detector, and the decay angle is more likely to be large, which translates into a larger delay for the photon. The fraction of events with a delayed photon arrival time initially rises as a function of $\tilde{\chi}_{1}^{0}$ lifetime, but falls as the fraction of $\tilde{\chi}_{1}^{0}$ 's decaying outside the detector begins to dominate. In the $\tilde{\chi}_{1}^{0}$ mass region considered $\left(65 \leq m_{\tilde{\chi}_{1}^{0}} \leq 150 \mathrm{GeV} / c^{2}\right)$, the acceptance peaks at a lifetime of around $5 \mathrm{~ns}$. The acceptance also depends on the mass as the boost effects are mitigated by the ability to produce high energy photons or $\mathbb{E}_{T}$ in the collision [10].

The total systematic uncertainty of $10 \%$ on the acceptance is dominated by the uncertainty on the mean of the $t_{c}^{\gamma}$ distribution (7\%) and on the photon ID efficiency (5\%). Other significant contributions come from uncertainties on 


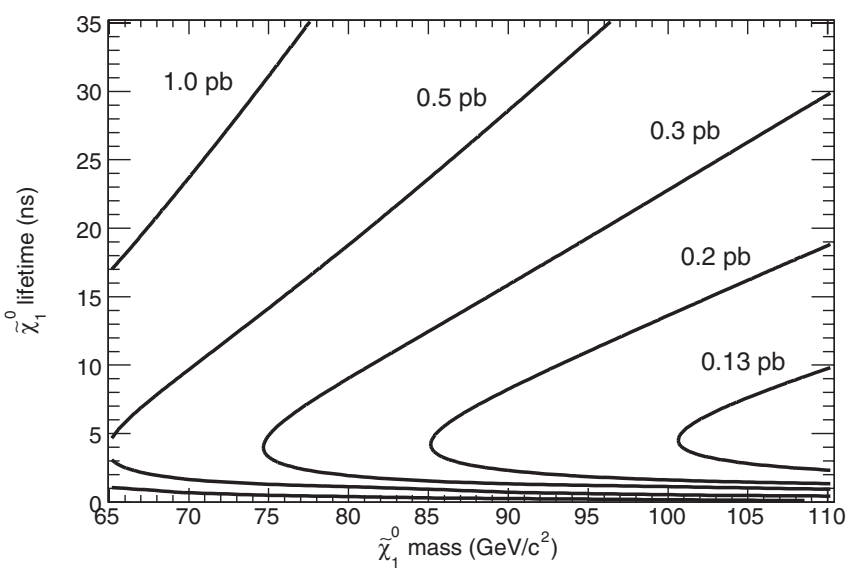

FIG. 2. The contours of constant $95 \%$ C.L. upper cross section limits for a GMSB model [9].

initial and final state radiation (3\%), jet energy measurement $(3 \%)$, and the parton distribution functions $(1 \%)$.

We determine the kinematic and $t_{c}^{\gamma}$ selection requirements that define the final data sample by optimizing the predicted cross section limit without looking at the data in the signal region. To compute the predicted $95 \%$ confidence level (C.L.) cross section upper limit [17], we combine the predicted GMSB signal and background estimates with the systematic uncertainties using a Bayesian method [18]. The predicted limits are optimized by simultaneously varying the selection requirements for $\mathbb{E}_{T}$, photon $E_{T}$, jet $E_{T}$, azimuth angle between the leading jet and $\mathscr{E}_{T}$ $\left(\Delta \phi\left(\mathbb{E}_{T}\right.\right.$, jet $\left.)\right)$, and $t_{c}^{\gamma}$. The $\Delta \phi\left(\mathscr{E}_{T}\right.$, jet $)$ requirement rejects events where the $\mathscr{E}_{T}$ is overestimated because of a poorly measured jet. While each point in the considered $\widetilde{\chi}_{1}^{0}$ lifetime vs mass space gives a slightly different optimization, we choose a single set of requirements because it simplifies the final analysis, while only causing a $<4 \%$ loss of sensitivity. The optimized requirements are summarized in Table I. The acceptance for the example model point is estimated to be $(6.3 \pm 0.6) \%$.

After all kinematic requirements, 508 events are observed in the data before the final signal region time requirement. Their $t_{c}^{\gamma}$ distribution is shown in Fig. 1. Our fit to the data outside the signal region predicts total backgrounds of $6.2 \pm 3.5$ from cosmic rays, $6.8 \pm 4.9$ from beam halo background sources, and the rest from the standard model with a measured wrong vertex fraction of $(0.5 \pm 0.2) \%$. Inside the signal region, $\{2,10\}$ ns, we predict $1.25 \pm 0.66$ events: $0.71 \pm 0.60$ from standard model, $0.46 \pm 0.26$ from cosmic rays, and $0.07 \pm 0.05$ from beam halo. Two events are observed in the data. Since the result is consistent with the no-signal hypothesis, we set predicted and observed limits on the $\widetilde{\mathcal{\chi}}_{1}^{0}$ lifetime and mass. Figure 2 shows the contours of constant $95 \%$ C.L. cross section upper limit which reflects the change in acceptance. The 95\% C.L. exclusion region, where the predicted production cross section at next-to-leading order [19] is

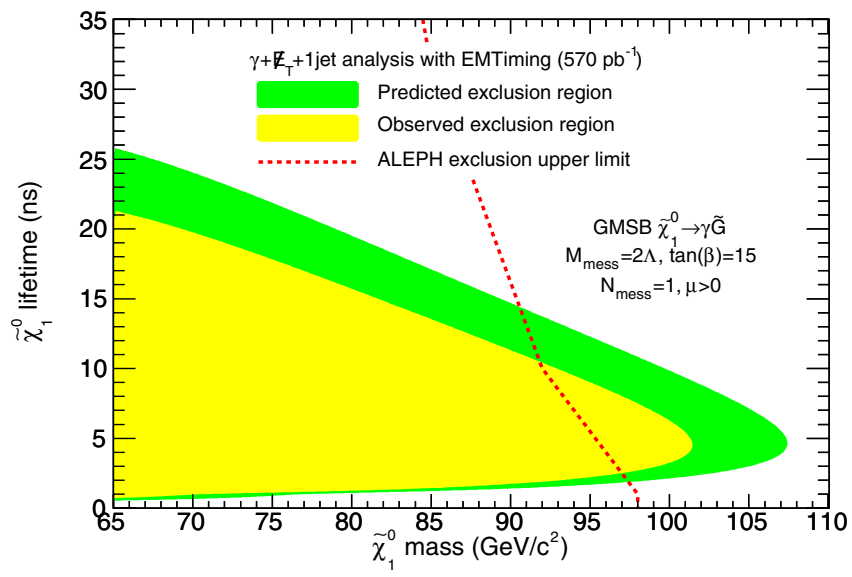

FIG. 3 (color online). The exclusion region at 95\% C.L. as a function of $\tilde{\chi}_{1}^{0}$ lifetime and mass for a GMSB model [9]. The predicted and the observed regions are shown separately and are compared to the most stringent published limit from LEP searches [8].

above the upper limit, is shown in Fig. 3, taking into account the uncertainties on the parton distribution functions $(6 \%)$ and the renormalization scale $(2 \%)$. These limits extend at large masses beyond those of LEP searches using photon "pointing" methods [8].

In conclusion, we have performed the first search for heavy, long-lived particles that decay to photons at a hadron collider using data collected with the EMTiming system at the CDF II detector. There is no excess of events beyond expectations. As our search strategy does not rely on event properties specific to GMSB models, we can exclude any delayed $\gamma+$ jet $+\not_{T}$ signal that would produce more than 5.5 events. We set cross section limits using a supersymmetric model with $\tilde{\chi}_{1}^{0} \rightarrow \gamma \tilde{G}$, and find a GMSB exclusion region in the $\tilde{\chi}_{1}^{0}$ lifetime vs mass plane with the world-best $95 \%$ C.L. lower limit on the $\tilde{\chi}_{1}^{0}$ mass of $101 \mathrm{GeV} / c^{2}$ at $\tau_{\tilde{\chi}_{1}^{0}}=5 \mathrm{~ns}$. By the end of run II, an integrated luminosity of $10 \mathrm{fb}^{-1}$ is possible for which we estimate a mass reach of $\simeq 140 \mathrm{GeV} / c^{2}$ at a lifetime of $5 \mathrm{~ns}$ by scaling the expected number of background events.

We thank the Fermilab staff and the technical staffs of the participating institutions for their vital contributions. This work was supported by the U.S. Department of Energy and National Science Foundation; the Italian Istituto Nazionale di Fisica Nucleare; the Ministry of Education, Culture, Sports, Science and Technology of Japan; the Natural Sciences and Engineering Research Council of Canada; the National Science Council of the Republic of China; the Swiss National Science Foundation; the A.P. Sloan Foundation; the Bundesministerium für Bildung und Forschung, Germany; the Korean Science and Engineering Foundation and the Korean Research Foundation; the Particle Physics and Astronomy Research Council and the Royal Society, UK; the Russian Foundation for Basic Research; the Comisión 
Interministerial de Ciencia y Tecnología, Spain; in part by the European Community's Human Potential Programme under Contract No. HPRN-CT-2002-00292; and the Academy of Finland.

${ }^{a}$ Visiting scientist from the University of Athens.

${ }^{\mathrm{b}}$ Visiting scientist from the University of Bristol.

${ }^{\mathrm{c}}$ Visiting scientist from the University Libre de Bruxelles.

${ }^{\mathrm{d}}$ Visiting scientist from Cornell University.

${ }^{\text {e}}$ Visiting scientist from the University of Cyprus.

${ }^{\mathrm{f}}$ Visiting scientist from the University of Dublin.

${ }^{\mathrm{g}}$ Visiting scientist from the University of Edinburgh.

${ }^{\mathrm{h}}$ Visiting scientist from the University of Heidelberg.

${ }^{\mathrm{i}}$ Visiting scientist from the Universidad Iberoamericana.

${ }^{\mathrm{j}}$ Visiting scientist from the University of Manchester.

${ }^{\mathrm{k}}$ Visiting scientist from the Nagasaki Institute of Applied Science.

${ }^{1}$ Visiting scientist from the University de Oviedo.

${ }^{\mathrm{m}}$ Visiting scientist from the University of London, Queen Mary College.

${ }^{\mathrm{n}}$ Visiting scientist from the University of California, Santa Cruz, USA.

${ }^{\circ}$ Visiting scientist from Texas Tech University, USA.

${ }^{\mathrm{p}}$ Visiting scientist from the University of California, Irvine, USA.

${ }^{\mathrm{q}}$ Visiting scientist from IFIC(CSIC-Universitat de Valencia).

[1] J. L. Feng, A. Rajaraman, and F. Takayama, Phys. Rev. D 68, 063504 (2003); M. J. Strassler and K. M. Zurek, arXiv:hep-ph/0605193.

[2] S. Ambrosanio et al., Phys. Rev. D 54, 5395 (1996); C. H. Chen and J. F. Gunion, Phys. Rev. D 58, 075005 (1998).

[3] F. Abe et al. (CDF Collaboration), Phys. Rev. Lett. 81, 1791 (1998); Phys. Rev. D 59, 092002 (1999).

[4] D. Acosta et al. (CDF Collaboration), Phys. Rev. D 71, 031104 (2005).

[5] V. Abazov et al. (D0 Collaboration), Phys. Rev. Lett. 94, 041801 (2005).
[6] P. Wagner, Ph.D. thesis, Texas A\&M University, 2007.

[7] D. Acosta et al. (CDF Collaboration), Phys. Rev. D 71, 032001 (2005).

[8] A. Heister et al. (ALEPH Collaboration), Eur. Phys. J. C 25, 339 (2002); also see M. Gataullin, S. Rosier, L. Xia, and H. Yang, arXiv:hep-ex/0611010; G. Abbiendi et al. (OPAL Collaboration), Proc. Sci., HEP2005 (2006) 346; J. Abdallah et al. (DELPHI Collaboration), Eur. Phys. J. C 38, 395 (2005).

[9] B. C. Allanach et al., Eur. Phys. J. C 25, 113 (2002). We use benchmark model 8 and take the messenger mass scale $M_{\text {mess }}=2 \Lambda, \tan (\beta)=15, \mu>0$ and the number of messenger fields $N_{\text {mess }}=1$. The $\widetilde{G}$ mass factor and the supersymmetry breaking scale $\Lambda$ are allowed to vary independently.

[10] D. Toback and P. Wagner, Phys. Rev. D 70, 114032 (2004).

[11] The distribution of the $p \bar{p}$ collisions has a standard deviation of $30 \mathrm{~cm}$ and $1.3 \mathrm{~ns}$ in $z_{i}$ and $t_{i}$, respectively.

[12] M. Goncharov et al., Nucl. Instrum. Methods Phys. Res., Sect. A 565, 543 (2006).

[13] The standard requirement, $\chi_{\text {CES }}^{2}<20$ [F. Abe et al. (CDF Collaboration), Phys. Rev. D 52, 4784 (1995)], has been removed because there is evidence that it is inefficient for photons that arrive with large incident angles relative to the face of the detector.

[14] A. Bhatti et al., Nucl. Instrum. Methods Phys. Res., Sect. A 566, 375 (2006). We use corrected jets reconstructed with a cone of 0.7 .

[15] T. Sjöstrand et al., Comput. Phys. Commun. 135, 238 (2001). We use version 6.216.

[16] We use the standard GEANT based detector simulation [R. Brun et al., CERN Report No. CERN-DD/EE/84-1, 1987] and add a parametrized EMTiming simulation.

[17] E. Boos, A. Vologdin, D. Toback, and J. Gaspard, Phys. Rev. D 66, 013011 (2002).

[18] J. Conway, CERN Yellow Book Report No. CERN 2000005,2000, p. 247 . We assume a flat prior in the production cross section.

[19] W. Beenakker et al., Phys. Rev. Lett. 83, 3780 (1999). 